\title{
PANDEMIA DA COVID-19: ALTERNATIVAS E ENTRAVES NO PROCESSO EDUCACIONAL
}

\author{
COVID-19 PANDEMIC: ALTERNATIVES AND \\ OBSTACLES IN THE EDUCATIONAL PROCESS
}

\section{PANDEMIA DE COVID-19: ALTERNATIVAS Y OBSTÁCULOS EN EL PROCESO EDUCATIVO}

\author{
Hevillyn Cecília Ventura Barbosa ${ }^{1}$ \\ Kalyne Araújo Bezerra ${ }^{2}$ \\ Maria Karoline Santos Lima ${ }^{3}$ \\ Maêlly Cristinne Ventura Barbosa ${ }^{4}$ \\ José Arthur Guimarães dos Santos ${ }^{5}$ \\ Elisângela Braga de Azevedo ${ }^{6}$
}

Como citar este artigo: Barbosa HCV, Bezerra KA, Lima MKS, Barbosa MCV, Santos JAG, Azevedo EB. Pandemia da COVID-19: alternativas e entraves no processo educacional. Rev baiana enferm. 2022;36:e38170.

Objetivo: identificar as implicações da pandemia da COVID-19 na educação de discentes do ensino superior da área da saúde e averiguar as metodologias de ensino adotadas pelas instituições para formação profissional. Método: revisão integrativa, descritiva, exploratória e qualitativa. Aplicados os descritores: "coronavirus infections", "pandemics" e "bealth training" na PubMed e "infecções por coronavirus", "pandemias" e "formação em saúde" no IBECS e na BVS. Selecionados 13 artigos. expostos em quadro síntese. Resultados: identificaram-se impossibilidade do encontro presencial, articulação de órgãos e instituições de ensino, cancelamento de estágios e aulas eletivas, encurtamento de estágios, antecipação de formatura e inserção de discentes na assistência; foram adotadas metodologias de ensino, como educação remota, aplicação de simulados e palestras, compactação das aulas em estágios. Considerações finais: mesmo com a pandemia da COVID-19 a educação superior da área da saúde continuou com as atividades acadêmicas por meio de ações remotas, visando a permanência do aprendizado.

Descritores: Infecções por Coronavírus. Educação Superior. Pandemia. Capacitação de Recursos Humanos em Saúde. COVID-19.

Objective: to identify the implications of the COVID-19pandemic in the education of higher education students in the bealth area and to investigate the teaching methodologies adopted by institutions for professional training. Method: integrative, descriptive, exploratory and qualitative review. The descriptors: "coronavirus infections", "pandemics" and "health training" in PubMed and "infecções por coronavirus", "pandemias" and "formação em saúde" at IBECS and VHL were applied. Selected 13 articles, exposed in summary chart. Results: the impossibility of the face-to-face meeting, articulation of teaching bodies and institutions, cancellation of internships and elective classes, shortening of internships, anticipation of graduation and insertion of students in care were identified; teaching methodologies were adopted, such as remote education, application of simulations and lectures, compaction of

Estudante de Enfermagem. Centro Universitário UNIFACISA. Campina Grande, Paraíba, Brasil. hevillynceciliav@gmail.com. https://orcid.org/0000-0002-2299-9869. Estudante de Enfermagem. Centro Universitário UNIFACISA. Campina Grande, Paraíba, Brasil. https://orcid.org/0000-000 I-7I $77-8950$.

Estudante de Enfermagem. Centro Universitário UNIFACISA. Campina Grande, Paraíba, Brasil. https://orcid.org/ 0000-000 I-8I 08-9980

Estudante de Psicologia. Centro Universitário UNIFACISA. Campina Grande, Paraíba, Brasil. https://orcid.org/0000-000 I-8397-545X.

Estudante de Enfermagem. Centro Universitário UNIFACISA. Campina Grande, Paraíba, Brasil. https://orcid.org/0000-0002-845I-8055.

Enfermeira. Doutora em Enfermagem. Professora do Centro Universitário UNIFACISA. Campina Grande, Paraíba, Brasil. https://orcid.org/0000-0002-9529-03 I6. 
classes in internships. Final considerations: even with the COVID-19 pandemic, higher education in the health area continued with academic activities through remote actions, aiming at the permanence of learning.

Descriptors: Coronavirus Infections. Education, Higher. Pandemic. Health Human Resource Training. COVID-19.

Objetivo: identificar las implicaciones de la pandemia de COVID-19 en la educación de los estudiantes de educación superior en el área de la salud e investigar las metodologías de enseñanza adoptadas por las instituciones para la formación profesional. Método: revisión integradora, descriptiva, exploratoria y cualitativa. Se aplicaron los descriptores: "coronavirus infections", "pandemics" y "health training" en PubMed y "infecções por coronavirus", "pandemias" y "formação em saúde" en IBECS y BVS. Selección de 13 artículos, expuestos en cuadro resumido. Resultados: se identificó la imposibilidad de la reunión presencial, articulación de cuerpos e instituciones docentes, cancelación de pasantías y clases electivas, acortamiento de pasantías, anticipación de graduación e inserción de estudiantes en cuidado; se adoptaron metodologías de enseñanza, como la educación a distancia, la aplicación de simulaciones y conferencias, la compactación de clases en pasantías. Consideraciones finales: incluso con la pandemia de COVID-19, la educación superior en el área de la salud continuó con actividades académicas a través de acciones remotas, apuntando a la permanencia del aprendizaje.

Descriptores: Infecciones por Coronavirus. Educación Superior. Pandemia. Capacitación de Recursos Humanos en Salud. COVID-19.

\section{Introdução}

Em meados de dezembro de 2019, declarações sobre a emergência de um surto do novo coronavírus na China teve grande repercussão e tornou-se tema de discussão em todo o mundo. Gradativamente, os demais países foram notificados sobre a possibilidade de infestação e disseminação do vírus, que teria um elevado grau de transmissibilidade e letalidade. Em março de 2020, além da China, começaram a surgir novos casos em outros países de forma exponencial. A Organização Mundial de Saúde (OMS) declarou emergência de saúde pública de interesse internacional, caracterizando a situação como uma pandemia ${ }^{(1)}$.

Pertencente à família Coronaviridae, o SARS-Cov-2 é o agente responsável por causar a COVID-19, patologia que pode desencadear diversos sintomas respiratórios, tendo potencial de variar desde um simples quadro gripal a uma Síndrome Respiratória Aguda Grave (SRAG) ${ }^{(2)}$.

A situação de crise vivenciada nos últimos meses, devido à referida pandemia, tem levado a população a reflexões que abrangem todos os aspectos da vida humana. Deste modo, tornou-se notório que os governantes de todo o mundo estão incansavelmente investindo em políticas públicas, ações e medidas que contenham os efeitos nocivos do vírus ao sistema biológico dos indivíduos. Além disso, o crescimento exponencial de casos positivos para a COVID-19, o aumento das internações em leitos de unidades de terapia intensiva e os números elevados de óbitos pelo vírus têm preocupado os órgãos responsáveis e a população, além de estar causando pânico entre os indivíduos, o que torna as medidas de precaução mais essenciais ${ }^{(3)}$.

Cabe evidenciar que, dentre as medidas reconhecidas pelo Ministério da Saúde, o distanciamento social e o autocuidado tornaram-se as prevenções mais eficazes no combate à doença, assim como em outros períodos de pandemia. Todavia, estas precauções trouxeram mudanças repentinas na rotina dos indivíduos, que se viram diante da necessidade de adaptar-se a um novo modo de viver e de trabalhar, em geral, nos próprios domicílios. O foco direcionou-se ao uso de dispositivos tecnológicos para interagir com a família e os amigos, nas atividades laborais, como no bome office e no ensino on-line. Este tornou-se uma nova forma para a continuidade das atividades acadêmicas $^{(4)}$, que precisaram ser revistas pelas instituições, pelos docentes e, principalmente, pelos discentes, receptores do produto final, resultando em alterações significativas nos comportamentos de ensino e aprendizagem ${ }^{(5)}$. 
Além disso, é inegável, que a quarentena causada pela COVID-19 ocasionou uma crise de nível global na saúde pública e mental. A Word Health Organization (WHO) define, como saudável, o indivíduo que se apresenta em bem-estar físico, mental e social, não sendo apenas a ausência de patologias ou enfermidades o único fator para estabelecer sua condição de saúde. Para tanto, o convívio em sociedade é um determinante poderoso para o desenvolvimento do ser humano e a manutenção de sua saúde ${ }^{(6)}$.

Foi nessa direção e observando a magnitude do problema, que o Conselho Nacional de Educação (CNE) lançou diretrizes que aprovaram um parecer tanto para a educação básica quanto para o ensino superior durante a pandemia. Assim, os sistemas de ensino foram autorizados a realizar ações de modo remoto, para o cumprimento da carga horária obrigatória ${ }^{(7)}$. Com isso, criou-se a possibilidade de desenvolver estudos on-line, para não prejudicar o processo de formação do acadêmico.

Todas essas mudanças possibilitaram aos gestores das instituições de ensino reinventarem as metodologias e os recursos pedagógicos aplicados ao centro de educação, desenvolvendo novas formas de aprendizado, de modo que se preservasse a fidedignidade dos conteúdos passados, bem como a qualidade do aprendizado. Sendo assim, o uso das metodologias remotas foi inserido nesse contexto com uma intensidade e velocidade impressionantes, mostrando-se como um desafio de extrema importância para os docentes, em especial os dos cursos de saúde, que tiveram de romper paradigmas e prover os discentes da sensação de imersão, navegação e interesse, mesmo em meio a um cenário virtual ${ }^{(8)}$.

Nessa direção, partiu-se do seguinte questionamento: Quais as implicações da pandemia da COVID-19 na educação de discentes do ensino superior e as alternativas adotadas pelas instituições da área de saúde presentes na literatura científica? Desse modo, teve-se como objetivos: identificar as implicações da pandemia da COVID-19 na educação de discentes do ensino superior da área da saúde e averiguar as metodologias de ensino adotadas pelas instituições para a formação profissional.

\section{Método}

Trata-se de uma revisão integrativa de caráter descritivo, exploratório com abordagem qualitativa. Para isso, seguiu-se as etapas propostas para uma revisão integrativa: identificação do tema e seleção da hipótese ou questão de pesquisa; estabelecimento dos critérios de inclusão e exclusão dos estudos; definição das informações a serem extraídas dos estudos; avaliação dos estudos incluídos (nível de evidência); interpretação dos resultados; e síntese do conhecimento ${ }^{(9)}$.

O traçado do tema e a elaboração da questão de pesquisa foram norteados pela estrutura do acrônimo PICo utilizada em pesquisas não clínicas, em que o $\mathrm{P}$ (paciente) trata-se dos acadêmicos, docentes e instituições de ensino, o I (interesse) refere-se às alternativas adotadas e o Co (contexto) alude à situação pandêmica vivenciada devido à COVID-19.

Posteriormente, realizou-se a busca na Biblioteca Virtual de Saúde (BVS) com os descritores "Infecções por coronavirus" AND "Pandemias" AND "Formação em saúde" (palavra-chave utilizada). $\mathrm{Na}$ BVS foram encontrados 14 artigos originados da base de dados Medical Literature Analysis and Retrieval System Online (MEDLINE). Foram critérios de inclusão: manuscritos no idioma inglês, de acordo com os resultados das bases de dados, publicados no último ano (2019 a 2020), disponíveis na íntegra. O critério de exclusão foi ser manuscrito duplicado. Após leitura de título e resumo, foram excluídas três publicações. Desse modo, foram selecionados oito artigos. Também se realizou a busca na plataforma Índice Bibliográfico de Ciências em Saúde (IBECS), aplicando os mesmos descritores e a palavra-chave, mas não foi obtido nenhum resultado.

Em seguida, os descritores e a palavra-chave foram aplicados na PubMed Central (PMC) associados ao operador booleano "AND": "Coronavirus infections" AND "Pandemics" AND "Health training" sendo obtido um total de 853 publicações. Após aplicação dos critérios de inclusão, ficaram 594 documentos científicos. Após leitura do título, restaram 24 artigos; 11, após leitura dos resumos; e, no final, foram selecionados 7 artigos. 
Dessa maneira, ao final da etapa de seleção, obteve-se um total de 16 artigos. Entretanto, três encontravam-se em mais de uma base de dados. Com isso, a amostra do presente estudo totalizou 13 artigos científicos. Levando em consideração os seis passos para a construção de uma revisão integrativa, realizou-se a classificação dos níveis de evidência seguindo o modo de GRADE, para melhor seriação da amostra.

A Figura 1 ilustra o processo de seleção dos artigos:

Figura 1 - Seleção dos artigos

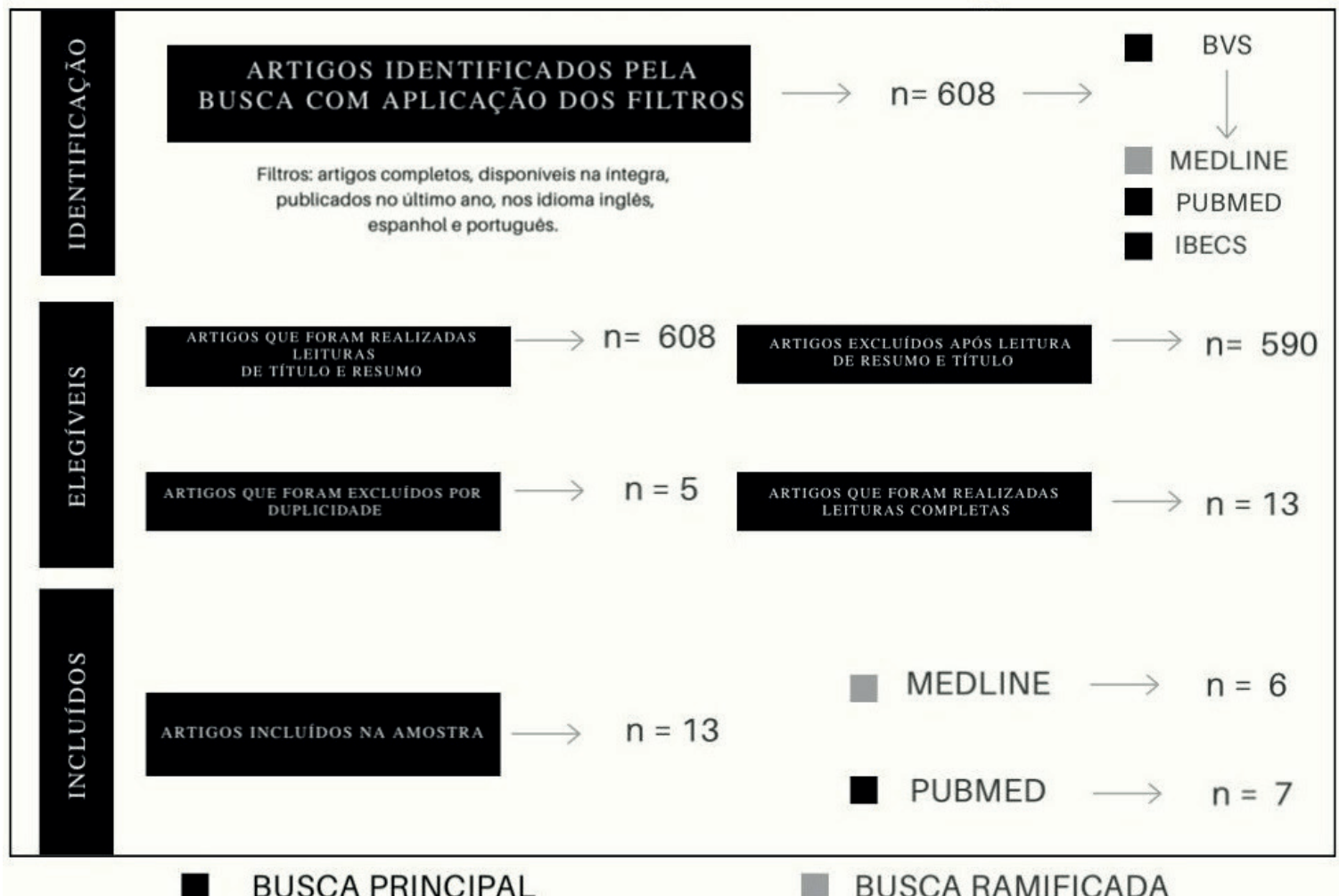

Fonte: Elaboração própria.

\section{Resultados}

Os dados foram organizados em um quadro de categorização dos trabalhos, levando em consideração critérios como: título, nível de evidência, país, curso e implicações da pandemia para a educação, assim como as alternativas adotadas. Logo após, na discussão, será empreendida a análise crítica do conteúdo trazido pelos artigos e citados, bem como a síntese dos achados.

Quadro 1 - Descrição dos estudos quanto ao título, nível de evidência, país, curso e implicações da pandemia em relação à educação e alternativas adotadas

\begin{tabular}{|c|c|c|c|c|}
\hline Título do estudo & $\begin{array}{l}\text { Nivel de } \\
\text { evidência }\end{array}$ & País & Curso & $\begin{array}{c}\text { Implicações/ alternativas } \\
\text { adotadas }\end{array}$ \\
\hline $\begin{array}{l}\text { The coronavirus } \\
\text { (COVID-19) pandemic: } \\
\text { Adaptations in medical } \\
\text { education }\end{array}$ & Nível II & $\begin{array}{l}\text { Reino } \\
\text { Unido }\end{array}$ & Medicina & $\begin{array}{l}\text { Os exames foram realizados } \\
\text { por meio da exposição de } \\
\text { vídeos e imagens; alguns } \\
\text { estudantes tiveram a opção de } \\
\text { adiantar os exames, fazê-los } \\
\text { de forma remota ou cancelar; } \\
\text { cancelamento dos } \\
\text { estágios eletivos. }\end{array}$ \\
\hline
\end{tabular}


Quadro 1 - Descrição dos estudos quanto ao título, nível de evidência, país, curso e implicações da pandemia em relação à educação e alternativas adotadas

(continuação)

\begin{tabular}{|c|c|c|c|c|}
\hline Título do estudo & $\begin{array}{l}\text { Nivel de } \\
\text { evidência }\end{array}$ & País & Curso & $\begin{array}{l}\text { Implicações/ alternativas } \\
\text { adotadas }\end{array}$ \\
\hline $\begin{array}{l}\text { The current effects of the } \\
\text { spread of COVID-19 in } \\
\text { learning environments } \\
\text { involving Japanese } \\
\text { college students: What } \\
\text { is the state of those } \\
\text { environments elsewhere } \\
\text { in the world? }\end{array}$ & Nível II & Japão & $\begin{array}{l}\text { Estudantes } \\
\text { universitários }\end{array}$ & $\begin{array}{l}\text { As aulas passaram a ser } \\
\text { on-line e a situação da } \\
\text { COVID-19 despertou } \\
\text { sentimento de desistência para } \\
\text { os estudantes, em decorrência } \\
\text { da situação financeira. }\end{array}$ \\
\hline $\begin{array}{l}\text { Impact of Sars-Cov-2 } \\
\text { And Its Reverberation in } \\
\text { Global Higher Education } \\
\text { And Mental Health }\end{array}$ & Nível II & Brasil & Universitários & $\begin{array}{l}\text { A conversão do ensino } \\
\text { presencial em ensino on-line } \\
\text { ocasionou estresse, ansiedade } \\
\text { e depressão nos estudantes. }\end{array}$ \\
\hline $\begin{array}{l}\text { The impact of the } \\
\text { COVID-19 pandemic on } \\
\text { final year medical students } \\
\text { in the United Kingdom: a } \\
\text { national survey }^{(12)}\end{array}$ & Nível IV & $\begin{array}{l}\text { Reino } \\
\text { Unido }\end{array}$ & Medicina & $\begin{array}{l}\text { Realização de testes on-line, } \\
\text { cancelamento ou encurtamento } \\
\text { de estágios. }\end{array}$ \\
\hline $\begin{array}{l}\text { Coalition for medical } \\
\text { education-A call to } \\
\text { action: A proposition to } \\
\text { adapt clinical medical } \\
\text { education to meet the } \\
\text { needs of students and } \\
\text { other healthcare learners } \\
\text { during COVID-19 }\end{array}$ & Nível V & $\begin{array}{l}\text { Estados } \\
\text { Unidos }\end{array}$ & Medicina & $\begin{array}{l}\text { Uso de plataformas como } \\
\text { Zoom e WebEx para palestras } \\
\text { e simulações, principalmente } \\
\text { para alunos que cursam do } 3^{\circ} \\
\text { ano de medicina em diante. }\end{array}$ \\
\hline $\begin{array}{l}\text { Pedagogical foundations } \\
\text { to online lectures in } \\
\text { health professions } \\
\text { education }\end{array}$ & Nível II & Austrália & $\begin{array}{l}\text { Estudantes } \\
\text { universitários }\end{array}$ & $\begin{array}{l}\text { As aulas on-line trouxeram } \\
\text { preocupações principalmente } \\
\text { relacionadas à absorção do } \\
\text { conteúdo, pela possibilidade } \\
\text { de estarem em outras } \\
\text { atividades simultaneamente. }\end{array}$ \\
\hline $\begin{array}{l}\text { Nepalese Medical } \\
\text { Students in the } \\
\text { COVID-19 Pandemic: } \\
\text { Ways Forward }\end{array}$ & Nível VII & Nepal & Medicina & $\begin{array}{l}\text { Formatura antecipada; alunos } \\
\text { postos para atuar na linha } \\
\text { de frente contra a COVID-19, } \\
\text { expondo-os a riscos de } \\
\text { contaminação, estresse físico e } \\
\text { psicológico. }\end{array}$ \\
\hline $\begin{array}{l}\text { Using Technology to } \\
\text { Maintain the Education } \\
\text { of Residents During the } \\
\text { COVID-19 Pandemic }\end{array}$ & Nível II & $\begin{array}{l}\text { Estados } \\
\text { Unidos }\end{array}$ & $\begin{array}{l}\text { Residência em } \\
\text { cirurgia }\end{array}$ & $\begin{array}{l}\text { Continuação, por meio de } \\
\text { plataformas on-line, com } \\
\text { simulação de procedimentos e } \\
\text { vídeos cirúrgicos. }\end{array}$ \\
\hline $\begin{array}{l}\text { Coronavirus Disease } \\
2019 \text { (COVID-19) and } \\
\text { Radiology Education- } \\
\text { Strategies for Survival }^{(17)} \\
\end{array}$ & Nível V & $\begin{array}{l}\text { Estados } \\
\text { Unidos }\end{array}$ & Radiologia & $\begin{array}{l}\text { Uso de tecnologias para ensino } \\
\text { didático e discussão de casos } \\
\text { por meio de artigos e envio de } \\
\text { imagens para melhor precisão. }\end{array}$ \\
\hline $\begin{array}{l}\text { COVID-19 pandemic: } \\
\text { Beyond medical } \\
\text { education in Brazil }^{(18)}\end{array}$ & Nível II & Brasil & $\begin{array}{l}\text { Medicina, } \\
\text { enfermagem, } \\
\text { fisioterapia e } \\
\text { farmácia }\end{array}$ & $\begin{array}{l}\text { Suspensão temporária das } \\
\text { aulas, pelas instituições } \\
\text { públicas, devido à } \\
\text { vulnerabilidade social dos } \\
\text { estudantes. }\end{array}$ \\
\hline
\end{tabular}


Quadro 1 - Descrição dos estudos quanto ao título, nível de evidência, país, curso e implicações da pandemia em relação à educação e alternativas adotadas

(conclusão)

\begin{tabular}{|l|l|l|l|l|}
\hline Título do estudo & $\begin{array}{c}\text { Nivel de } \\
\text { evidência }\end{array}$ & País & \multicolumn{1}{c|}{ Curso } & \multicolumn{1}{c|}{$\begin{array}{c}\text { Implicações/ alternativas } \\
\text { adotadas }\end{array}$} \\
\hline $\begin{array}{l}\text { Practical approaches } \\
\text { to pedagogically rich } \\
\text { online tutorials in health } \\
\text { professions education }\end{array}$ & Nível II & Austrália & $\begin{array}{l}\text { Estudantes } \\
\text { universitários }\end{array}$ & $\begin{array}{l}\text { A sala de aula invertida } \\
\text { permitiu que os próprios } \\
\text { alunos se tornassem os } \\
\text { protagonistas, além de } \\
\text { promover melhor interação } \\
\text { entre alunos e professores. } \\
\text { Foi uma estratégia de ensino } \\
\text { para atrair a atenção dos } \\
\text { discentes no ensino on-line. }\end{array}$ \\
\hline $\begin{array}{l}\text { Medical genetics } \\
\text { education in the midst of } \\
\text { the COVID-19 pandemic: } \\
\text { Shared resources }\end{array}$ & Nivel II & $\begin{array}{l}\text { Estados } \\
\text { Unidos }\end{array}$ & Medicina & $\begin{array}{l}\text { Por meio de imagens, os } \\
\text { estudantes anonimamente } \\
\text { indicavam anormalidades pelo } \\
\text { bate-papo da plataforma Zoom. }\end{array}$ \\
\hline $\begin{array}{l}\text { Adapting to a new reality: } \\
\text { COVID-19 coronavirus } \\
\text { and online education in } \\
\text { the health professions }\end{array}$ & Nível II & Austrália & $\begin{array}{l}\text { Estudantes } \\
\text { universitários }\end{array}$ & $\begin{array}{l}\text { Novos desafios na implantação } \\
\text { de aulas on-line, como } \\
\text { o bem-estar, a saúde, a } \\
\text { segurança e a disciplina. Foi } \\
\text { importante a realização de } \\
\text { videoconferências, para melhor } \\
\text { interação. }\end{array}$ \\
\hline
\end{tabular}

Fonte: Elaboração própria.

\section{Discussão}

Após avaliação dos manuscritos, foram identificados trabalhos de nível de evidência II, IV, V e VII. Também se observou variação na escala geográfica, apresentando-se nos Estados Unidos, Brasil, Japão, Reino Unido, Austrália e Nepal, com amostras populacionais dos cursos de medicina, enfermagem, radiologia, fisioterapia, farmácia e residentes em cirurgia. Deste modo, diante dos desafios impostos pelas circunstâncias, foi possível observar certa heterogeneidade nos meios utilizados por estudantes e instituições para facilitar a aprendizagem por meio eletrônico.

A COVID-19 teve sua origem na cidade Wuhan, na China. Desde seu aparecimento, os governos recomendaram que os cidadãos utilizassem o sistema remoto para suas atividades de ofício e ensino. Assim, devido à queda verificada na economia, muitas pessoas tiveram sua renda reduzida. Tal fato desencadeou, nos discentes, pensamentos de desistência ou mesmo descontinuidade de seus cursos, pois, diante do processo de mudança que passaram a vivenciar, acreditaram na impossibilidade de se sustentarem financeiramente no decorrer da pandemia. Por isso, tornou-se necessária a articulação das entidades sociais, para chegarem a alternativas e soluções para o ramo de ensino, visto que uma queda na produção científica de um país significa também um regresso às próprias soluções do quadro epidemiológico atual ${ }^{(10)}$.

Assim, considerando essa realidade, muitas instituições de ensino mantiveram-se fechadas, para garantir a segurança de discentes, docentes e servidores. Diante dessa realidade, o ensino on-line tornou-se uma alternativa para a aprendizagem, embora apresente algumas dificuldades. Muitos acadêmicos não possuíam acesso à internet e também não detinham ferramentas tecnológicas para assistir às aulas, devido às desigualdades sociais e econômicas dos países, sobretudo no Brasil. Outros aspectos pouco explorados na literatura existente sobre os impactos da COVID-19 são os problemas psicológicos que poderão decorrer dessa parada de atividades educacionais. A ansiedade e o estresse, por exemplo, são sentimentos compartilhados 
pela comunidade e, portanto, a paralisação de atividades e a incerteza quanto ao quadro epidemiológico podem ser prejudiciais ${ }^{(11)}$.

Estudos realizados citam a utilização de plataformas on-line, como Zoom e WebEx, para execução do ensino em saúde, assim como a aplicabilidade de simulados e palestras para os discentes ${ }^{(12-13)}$. Ainda destacando a prática de compactação das aulas em estágios, explanaram a implantação dos exames das disciplinas, por meio da exposição de vídeos e imagens ${ }^{(14)}$.

Como alternativas, diversos métodos de ensino podem ser aplicados de forma on-line. Entretanto, é necessário que o conteúdo abordado tenha como objetivo envolver o discente e tornar o processo de aprendizagem mais acessível. Isto é, a utilização de metodologias ativas emerge como um pilar fundamental, para que haja sucesso nas aulas. Dessa forma, o período pandêmico vivenciado desafiou os envolvidos no processo educacional e convidou-os a aplicar o conhecimento adquirido mediante abordagens didáticas e remotas. Com isso, promoveu um ambiente de aprendizagem mais confortável para os discentes ${ }^{(15)}$.

Desse modo, diante da grande urgência perante o quadro epidemiológico da COVID-19 e da necessidade de atuação dos professores nessa realidade, foi necessário pensar sobre tais medidas no ensino em saúde ${ }^{(8)}$. Assim, muitas instituições de ensino cancelaram seus estágios e aulas práticas e precisaram adaptar-se à realidade remota, com a utilização de ferramentas on-line, indispensáveis no cenário epidemiológico, pois é difícil imaginar a retomada das atividades. À vista disso, essa crise poderá servir de impulso para o planejamento de um ensino profissional mais inovador e adaptativo ao aprendizado contemporâneo ${ }^{(13)}$.

No Reino Unido, por exemplo, por meio de uma investigação com acadêmicos de medicina do último ano de curso, buscou-se compreender os principais impactos causados pela situação pandêmica no ensino. Inferiu-se que muitos estágios e aulas eletivas foram cancelados, diante da impossibilidade de realização, mas a maioria deles também concordou que o auxílio em hospitais, diante dessa realidade, seria de grande importância para o aprendizado. Assim, compreendeu-se que, manter as atividades, tal como acontecia antes do quadro epidemiológico atual, seria prejudicial e, portanto, seria mais indicada a utilização de atividades remotas, como cursos, palestras e estágios voluntários ${ }^{(12)}$.

Em pesquisa realizada no Nepal, observou-se que os discentes de medicina tiveram sua formatura antecipada e outros foram direcionados para trabalhar na linha de frente da COVID $19^{(15)}$.

Ficou evidente que as universidades passaram a adequar-se à nova realidade e isso, em grande parte, deu-se por meio de ferramentas on-line. Portanto, estudo afirmou que o ensino de residentes iria ser prejudicado se ficasse totalmente parado, uma vez que a formação de mais profissionais da saúde estaria em risco. Os autores ressaltaram ainda que, por mais que não se comparasse ao ensino prático, as universidades precisariam adaptar-se à aprendizagem remota, por meio do uso de videoconferências, aulas on-line e até mesmo a telemedicina ${ }^{(16)}$.

Em outro estudo, observou-se que, além do compromisso de manter a efetividade e qualidade do serviço prestado em radiologia aos pacientes, frente à pandemia da COVID-19, seria necessário também garantir a continuidade da formação, diante da necessidade do mercado de trabalho. Assim, constatou-se que seria de fundamental importância a implementação e utilização das ferramentas remotas. Tais mudanças foram possíveis por meio da consistência e continuação do aprendizado clínico baseado em discussões de casos clínicos e debates em plataformas on-line e da continuidade da aprendizagem com uma perspectiva mais criativa, atendendo às necessidades educacionais. Além disso, a utilização de banco de dados virtual, para análise de casos, e a interdisciplinaridade de áreas e papéis foram recursos que as universidades passaram a utilizar ${ }^{(17)}$.

Nesse sentido, compreendeu-se que a pandemia da COVID-19 configurou uma nova realidade de vida para os indivíduos, uma vez que tiveram de adaptar-se obrigatoriamente às medidas de distanciamento. Sendo assim, o sistema 
de ensino também precisou adequar-se às modalidades remotas, visto que o encontro presencial tornou-se impossibilitado ${ }^{(18)}$.

Neste estudo, identificou-se ainda que, principalmente em países emergentes, com economias subdesenvolvidas, houve uma preocupação com a formação profissional em geral, tendo em vista o quadro vivenciado, que se mostrou bastante adverso. Portanto, notou-se que o Brasil, por ter maior parte de sua formação centrada em instituições públicas que, por vezes, apresentam déficit estrutural para o ensino remoto, poderia sofrer com a pouca adaptabilidade e escassez de recursos para medidas contingentes durante a pandemia ${ }^{(18)}$.

Ademais, pesquisa observou muitas dificuldades de adaptação no ensino remoto, visto que os educadores não podem utilizar métodos usados em aulas presenciais para captar a atenção do discente sobre a temática abordada. Nas aulas on-line, o aluno fica sentado, com dispositivo móvel e inúmeras distrações que competem por atenção. Então, percebe-se que há um prejuízo na capacidade de o educador desenvolver um relacionamento com os acadêmicos, pois isso faz com que a identificação das motivações, dificuldades e experiências não estejam explícitas como ocorre nas discussões presenciais ${ }^{(19)}$.

A identificação de grande número de países afetados pelas mudanças consequentes da situação pandêmica revela os déficits nos sistemas de saúde de países com escassez de recursos. Por isso, muitos tiveram que reinventar suas formas de transmissão de ensino e construção de conhecimento, tendo em vista a realidade vivenciada. Ficou claro, contudo, que muitas medidas criativas também emergiram dos próprios discentes. Como exemplo, cita-se o caso do Nepal, um dos países, até o momento, menos afetado, mas que a posteriori poderia sofrer uma epidemia, também articulou discentes e Conselhos Éticos, para atuação de prevenção e promoção em saúde, por meio de medidas colaborativas de diferentes entidades administrativas, visando a diminuição do impacto do vírus no país ${ }^{(15)}$.
Como visto, no decorrer da crise pandêmica, muitos aspectos da vida moderna sofreram alterações e com o sistema educacional global não poderia ser diferente. Foi necessário reinventar-se e desenvolver opções de ensino ${ }^{(20)}$. A educação remota ganhou espaço e mostrou-se eficaz tanto quanto a forma convencional. Apesar de a aprendizagem presencial permanecer como um componente importantíssimo na formação acadêmica e curricular, tal modalidade pôde tornar-se mais uma opção viável e eficaz durante esse período. É válido ressaltar que o conteúdo e as metodologias aplicadas devem cumprir as exigências das autoridades de saúde e da educação, bem como seguir o plano de ensino designado para cada área ${ }^{(13)}$.

Com este estudo, acreditou-se que o processo adaptativo teve repercussões na vida das pessoas, principalmente no que tange ao distanciamento social. Todavia, vale salientar que o prolongamento das medidas contra o retorno das aulas presenciais pode ser prejudicial aos indivíduos enclausurados em suas residências ${ }^{(21)}$. Nesse sentido, a nova realidade poderá implicar em impactos na saúde mental dos discentes, tendo em vista as incertezas vivenciadas na atual conjuntura, que podem implicar na piora de quadros ansiosos e depressivos, por serem considerados os transtornos mais recorrentes nesse público ${ }^{(11)}$.

Tendo em vista o tipo de estudo, foram observadas algumas limitações. Dentre elas, a que mais se destacou foi a escassez de manuscritos nas bases de dados, para subsidiar uma amostra mais ampla e complexa. Tal fato ocorreu por tratar-se de um assunto recente e inesperado, tanto para a população em geral quanto para pesquisadores da área.

Todavia, a pesquisa possibilitou a análise dos diversos métodos educacionais adotados por diferentes países, compilando alternativas que podem ser utilizadas por instituições de outras nacionalidades. Por se tratar de um estudo integrativo, possibilitou a análise de vários estudos das mais diversas abordagens metodológicas, servindo como base complementar para futuros estudos com níveis de evidência mais elevados. 


\section{Considerações finais}

O cenário ocasionado pela pandemia da COVID-19 e a situação emergencial de adaptação da formação em saúde, que se baseia tanto na continuidade das atividades acadêmicas quanto na exiguidade da formação, sentiu-se a necessidade da articulação de vários órgãos e instituições para traçar ações remotas de ensino, visando a permanência do aprendizado, de modo a evitar o atraso na formação profissional.

As alternativas implementadas em diversos países foram inúmeras. As plataformas digitais foram as mais citadas nos manuscritos, variando entre opções mais dinâmicas e inovadoras e métodos antes utilizados de forma complementar no processo de formação presencial.

Outro ponto identificado nesta investigação foi que a pandemia possibilitou a inserção de alguns discentes da área de saúde na assistência, ajudando no processo de cuidado, ora emergencial, dos pacientes acometidos pela COVID-19, seja na linha de frente, seja na atenção primária, secundária ou terciária à saúde.

As mudanças metodológicas, mediante a utilização de plataformas on-line, como Zoom e WebEx, para execução do ensino em saúde, assim como a aplicabilidade de simulados e palestras para os discentes, a prática de compactação das aulas em estágios e a implantação dos exames das disciplinas, por meio da exposição de vídeos e imagens, além de benefícios, trouxeram também reflexões acerca das consequências psicológicas que se acentuaram e, por vezes, relacionaram-se ao quadro de mudanças que vieram à tona e estavam associadas à COVID-19, como foi o caso do distanciamento social, adesão total dos discentes a tal metodologia, encurtamento dos estágios, dentre outros questionamentos, segundo a literatura abordada.

Tendo em vista tais achados, percebeu-se a necessidade de se realizar mais estudos de cunho qualitativo e quantitativo para uma síntese mais ampla e sólida de tais itens citados nos primeiros artigos produzidos, além de ser necessária a identificação dos mais diversos modos de abordagens metodológicas das instituições e órgãos. Além do mais, é preciso que se tenha maior entendimento das consequências positivas e negativas de tal adaptação, de forma mais abrangente e concisa, que possam favorecer e contribuir para um refinamento do processo adaptativo e complementar no aprendizado em saúde.

\section{Colaborações:}

1 - concepção, projeto, análise e interpretação dos dados: Hevillyn Cecília Ventura Barbosa e Kalyne Araújo Bezerra;

2 - redação do artigo e revisão crítica relevante do conteúdo intelectual: Hevillyn Cecília Ventura Barbosa, Maria Karoline Santos Lima, Maêlly Cristinne Ventura Barbosa e José Arthur Guimarães dos Santos;

3 - aprovação final da versão a ser publicada: Elisângela Braga de Azevedo.

\section{Referências}

1. Brasil. Ministério da Saúde. Resposta nacional e internacional de enfrentamento ao novo coronavírus [Internet]. Brasília (DF); 2019 [cited 2020 Jul 12]. Available from: https://coronavirus. saude.gov.br/linha-do-tempo

2. Lana RM, Coelho FC, Gomes MFC, Cruz OG, Bastos LS, Villela DAM, et al. Emergência do novo coronavírus (SARS-CoV-2) e o papel de uma vigilância nacional em saúde oportuna e efetiva. Cad Saúde Pública. 2020;36(3):1-5. DOI: 10.1590/0102-311X00019620

3. Cruz RM, Borges-Andrade JE, Moscon DCB, Micheletto MRD, Esteves GGL, Delben PB, et al. COVID-19: emergência e impactos na saúde e no trabalho. Rev Psicol, Organ Trab. 2020;20(2):1-2. DOI: 10.17652/rpot/2020.2.editorial

4. Brasil. Ministério da Educação. Educação Superior a Distância [Internet]. Brasília (DF); 2020 [cited 2020 Jul 12]. Available from: http:// portal.mec.gov.br/instituicoes-credenciadas/ educacao-superior-a-distancia

5. Meo SA, Abukhalaf AA, Alomar AA, Sattar K, Klonoff DC. COVID-19 Pandemic: Impact of Quarantine on Medical Students' Mental Wellbeing and Learning Behaviors. Pak J Med Sci. 2020;36(COVID19-S4):S43-8. DOI: 10.12669/ pjims.36.COVID19-S4.2809 
6. World Health Organization. Mental health [Internet]. Genebra (CHE); 2019 [cited 2020 Jul 16]. Available from: https://www.who.int/news-room/ facts-in-pictures/detail/mental-health

7. Brasil. Ministério da Saúde. Saúde anuncia orientações para evitar a disseminação do coronavírus [Internet]. Brasília (DF); 2020 [cited 2020 Nov 13]. Available from: https://www.saude. gov.br/noticias/agencia-saude/46540-saudeanuncia-orientacoes-para-evitar-a-disseminacaodo-coronavirus

8. Alsafi Z, Abbas A-R, Hassan A, Ali MA. The coronavirus (COVID-19) pandemic: Adaptations in medical education. Int J Surg. 2020;78:64-5. DOI: $10.1016 /$ j.ijsu.2020.03.083

9. Sousa LMM, Marques-Vieira CMA, Severino SSP, Antunes AV. A metodologia de revisão integrativa da literatura em enfermagem. RIE [Internet]. 2017 Nov [cited 2020 Jul 10]:17-26. Available from: https://www.researchgate.net/ publication/321319742_Metodologia_de_Revisao_ Integrativa_da_Literatura_em_Enfermagem

10. Inoue $\mathrm{K}$, Takeshita $\mathrm{H}$, Ohira $\mathrm{Y}$. The current effects of the spread of COVID-19 in learning environments involving Japanese college students: What is the state of those environments elsewhere in the world? Int Maritime Health. 2020;71(2):150. DOI: $10.5603 /$ IMH.2020.0027

11. Araújo FJO, Lima LSA, Cidade PIM, Nobre CB, Rolim Neto ML. Impact Of Sars-Cov-2 And Its Reverberation In Global Higher Education And Mental Health. Psychiatry Res. 2020;288:112977. DOI: $10.1016 /$ j.psychres.2020.112977

12. Choi B, Jegatheeswaran L, Minocha A, Alhilani M, Nakhoul M, Mutengesa E. The impact of the COVID-19 pandemic on final year medical students in the United Kingdom: a national survey. BMC Med Educ. 2020;20:206. DOI: 10.1186/ s12909-020-02117-1

13. Newman NA, Lattouf OM. Coalition for medical education - A call to action: A proposition to adapt clinical medical education to meet the needs of students and other healthcare learners during COVID-19. J Card Surg. 2020;35(6):1174-5. DOI: $10.1111 /$ jocs. 14590

14. Seymour-Walsh AE, Weber A, Bell A. Pedagogical foundations to online lectures in health professions education. Rural Remote Health. 2020;20(2):6038. DOI: $10.22605 /$ RRH 6038

15. Guragai M. Nepalese Medical Students in the COVID-19 Pandemic: Ways Forward. JNMA J Nepal Med Assoc. 2020;58(225):352-4. DOI: 10.31729 / jnma.4924

16. Chick RC, Clifton GT, Peace KM, Propper BW, Hale DF, Alseidi AA, et al. Using Technology to Maintain the Education of Residents During the COVID-19 Pandemic. J Surg Educ. 2020;77(4):729-32.DOI: 10.1016/j.jsurg.2020.03.018

17. Slanetz PJ, Parikh U, Chapman T, Motuzas CL. Coronavirus Disease 2019 (COVID-19) and Radiology Education - Strategies for Survival. J Am Coll Radiol. 2020;17(6):743-5. DOI: 10.1016/ j.jacr.2020.03.034

18. Carvalho VO, Conceição LSR, Gois Jr MB. COVID-19 pandemic: Beyond medical education in Brazil. J Card Surg. 2020;35(6):1170-1. DOI: 10.1111/jocs. 14646

19. Seymour-Walsh AE, Weber A, Bell A. Practical approaches to pedagogically rich online tutorials in health professions education. Rural Remote Health. 2020;20(2):6045. DOI: 10.22605/RRH6045

20. Regier DS, Smith WM. Medical genetics education in the midst of the COVID-19 pandemic: Shared resources. Am J Med Genet A. 2020;182(6):1302-8. DOI: 10.1002/ajmg.a.61595

21. Seymour-Walsh AE, Bell A, Weber A, Smith T. Adapting to a new reality: COVID-19 coronavirus and online education in the health professions. Rural Remote Health. 2020;20(2):6000. DOI: 10.22605/RRH6000

Recebido: 31 de julho de 2020

Aprovado: 27 de maio de 2021

Publicado: 25 de janeiro de 2022

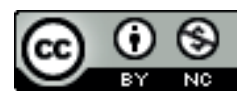

A Revista Baiana de Enfermagem utiliza a Licença Creative Commons - Atribuição-NãoComercial 4.0 Internacional.

https://creativecommons.org/licenses/by-nc/4.0/

Este artigo é de acesso aberto distribuído sob os termos da Licença Creative Commons (CC BY-NC).

Esta licença permite que outros remixem, adaptem e criem a partir do seu trabalho para fins não comerciais. Embora os novos trabalhos tenham de lhe atribuir o devido crédito e não possam ser usados para fins comerciais, os usuários não têm de licenciar esses trabalhos derivados sob os mesmos termos. 